\section{Comparación de la reacción inflamatoria inducidla por implantes faciales de silicona y politetrafluoroetileno. Estudio en conejos}

\section{Comparison of the inflammatory reaction induced by facial implants os silicone and polytetrafluoroethylene. Study in rabbits}

Fausto Viterbo*, María Aparecida C. DOMINGUES** Ryane S. BROCK ${ }^{\star \star \star}$, Eduardo FONSECA ${ }^{\star \star \star \star}$

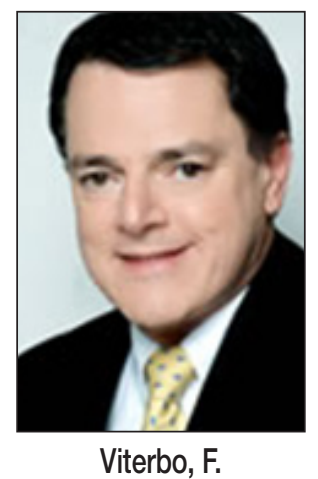

Resumen

Introducción y Objetivo. Existen diferentes tipos de injertos autólogos y materiales aloplásticos para el tratamiento de las diversas deformidades faciales. El politetrafluoroetileno (PTFE) tiene varias ventajas debido a su propiedad hidrofóbica, que induce menor reacción inflamatoria.

Presentamos un estudio que evalúa y compara la reacción inflamatoria inducida por los implantes faciales de silicona y de PTFE.

Material y Método. Colocamos fragmentos de implantes de silicona y de PTFE en orejas de conejos mediante incisión y disección de un bolsillo subcutáneo. Realizamos análisis histológico a las 8 semanas: tinción de las muestras con hematoxilina / eosina y calificación del grado de reacción inflamatoria crónica, presencia de neutrófilos, linfocitos, eosinófilos, neoangiogénesis, fibroblastos y edema, presencia o no de hemorragia y valoración de la cicatriz. Hicimos la recolección de muestras para análisis microbiológico y evaluación de la presencia de hematoma y absceso en el momento del sacrificio.

Resultados. La prevalencia de abscesos en el sacrificio, el hematoma y el edema durante las primeras semanas, fueron significativamente menores $(\mathrm{p}<0.05)$ en el grupo de PTFE.

Conclusiones. El PTFE indujo reacción inflamatoria crónica al igual que la silicona, pero con menos absceso, edema y formación de hematomas.
Keywords Silicon, Polytetrafluoroethylene, Facial prostheses, Facial implants.

Background and Objective. The treatment for many facial deformities uses many kinds of autologous grafts or alloplastic materials. Polytetrafluoroethylene (PTFE) has several advantages due to its hydrophobic properties, inducing less inflammatory reaction.

Our study evaluates and compares the inflammatory reaction induced by silicone and PTFE stripes.

Methods. Fragments of silicone and PTFE implants were placed in rabbits ears using an incision and a subcutaneous gap. The histological analysis was made 8 weeks later. The samples were stained with hematoxylin/eosin and classified as chronic inflammatory reaction graduation, the presence of neutrophils, lymphocytes, eosinophils, neoangiogenesis, fito microbiological analysis and evaluation of bruise and abscess were collected at the moment of sacrifice.

Results. Prevalence of abscess at sacrifice and hematoma during the first weeks were significantly higher $(p>0.05)$ in the silicon group. tion as the silicon but with less abscess and hematoma formation. broblasts, edema and presence of bleeding and scar. Samples

Conclusions. PTFE induced as much inflammatory reac-
Palabras clave Silicona, Politetrafluoroetileno, Prótesis faciales, Implantes faciales.
Nivel de evidencia científica
5 c Diagnóstico Recibido [esta versión] Aceptado
30 enero/2019

\section{Level of evidence Received [this version] Accepted}

Conflicto de intereses: los autores declaran no tener ningún interés financiero relacionado con el contenido de este artículo. Financiación: No hubo fuentes externas de financiación para este trabajo. 


\section{Introducción}

La etiología de las deformidades faciales puede ser congénita o adquirida. Las adquiridas se deben a traumatismos, infecciones, resecciones oncológicas o aparecen como secuela de radioterapia. ${ }^{(1)}$ Por lo que se refiere a las lesiones de causa traumática, han ido en aumento debido a los accidentes automovilísticos, entre los que el 24.4\% de los traumatismos faciales se produce en accidentes de motocicleta. $^{(2)}$

Muchas de estas lesiones causan deformidades cutáneas y óseas. Para su tratamiento podemos utilizar injertos autólogo de tejido (grasa o hueso) o implantar materiales aloplásticos tales como la silicona y los acrílicos. El injerto de grasa tiene muchas ventajas: es un material autólogo y provoca reacción inflamatoria mínima; sin embargo, requiere anestesia en el sitio donante y receptor y la absorción del tejido trasplantado es impredecible. ${ }^{(3)}$

En cuanto a los materiales aloplásticos, como la silicona, el politetrafluoroetileno (PTFE) y los acrílicos, tienen ventajas tales como: no requieren un sitio donante, lo que reduce el tiempo de procedimiento quirúrgico al eliminar la toma del injerto, su suministro es ilimitado si fuera necesario y permiten la opción de ser confeccionados individualmente para cada paciente.

Entre los materiales aloplásticos, la silicona, que se suministra en diferentes tamaños y formas ya sean láminas, bloques o gel envuelto por una cubierta también de silicona, es el más empleado y principalmente en este último formato. Aparte de la silicona, el uso de la PTFE está aumentando rápidamente en válvulas cardiacas de sustitución, en reconstrucción de la pared abdominal y en implantes faciales. ${ }^{(4)}$ Además, algunos estudios afirman que provoca menos reacción inflamatoria que la silicona. ${ }^{(5-7)}$

El objetivo del presente estudio es evaluar y comparar la reacción inflamatoria inducida por las láminas de silicona y PTFE implantadas en el tejido subcutáneo de conejos.

\section{Material y método}

Llevamos a cabo un estudio experimental en el Laboratorio de Cirugía Experimental del Departamento de Cirugía y Ortopedia de la Facultad de Medicina de Botucatu (FMB), UNESP, Brasil, tras aprobación por parte del Comité Ético de Experimentación Animal bajo el protocolo 1061/2013.

Empleamos un total de 10 conejos machos con unos 70 días de vida y 2.000 gr. de peso. Los animales fueron sometidos al procedimiento quirúrgico bajo anes- tesia con ketamina $35 \mathrm{mg} / \mathrm{kg}$ y xilazina $5 \mathrm{mg} / \mathrm{kg}$ intramuscular, según necesidad. Todo el material utilizado fue estéril para evitar la contaminación y los animales no recibieron antibióticos durante el período de observación.

En cada animal implantamos una banda de silicona de $60 \mathrm{~mm}$ de longitud, $25 \mathrm{~mm}$ de anchura y $12 \mathrm{~mm}$ de espesor $\left(\mathrm{FAGA}^{\circledR}\right.$ Industria de Equipamientos e Instrumentos Médico-Hospitalarios LTDA-ME, Bauru, Brasil) y una banda de PTFE de $60 \mathrm{~mm}$ de longitud, $18 \mathrm{~mm}$ de anchura y $0,5 \mathrm{~mm}$ de espesor $\left(\mathrm{FAGA}^{\circledR}\right.$ Industria de Equipamientos e Instrumentos Médico-Hospitalarios LTDA-ME, Bauru, Brasil) Colocamos los implantes en diferentes orejas de los animales de experimentación ordenadas al azar: un fragmento de silicona en una oreja y un fragmento de PTFE en la oreja contralateral. La definición de derecha o izquierda para cada fragmento se hizo por sorteo.

Practicamos una incisión de $1 \mathrm{~cm}$ de longitud sobre la cara interna de cada oreja; disecamos el tejido subcutáneo con el fin de crear un bolsillo de $2 \mathrm{~cm}$ de longitud y $2 \mathrm{~cm}$ de anchura en el que colocamos los implantes. Suturamos las incisiones con puntos simples usando monofilamento de nylon 5.0 y administramos una dosis intramuscular de tramadol de $5 \mathrm{mg} / \mathrm{kg}$.

Durante el postoperatorio, los animales fueron evaluados diariamente y ante cualquier signo de dolor o movilidad reducida administramos una dosis de tramadol 5 $\mathrm{mg} / \mathrm{kg}$ intramuscular para analgesia según la rutina de la FMB - vivero UNESP.

Tras el procedimiento, los animales se mantuvieron en jaulas individuales diseñadas para conejos, con alimentos y agua ad libitum y con ciclos de luz-oscuridad respetando el ritmo circadiano. La observación se mantuvo semanalmente durante 2 meses, evaluando edema, hiperemia, formación de abscesos y adherencias superficiales o profundas. Después de ese período, fueron sacrificados por sobredosis de anestesia con ketamina $150 \mathrm{mg} / \mathrm{kg}$ y xilazina $15 \mathrm{mg} / \mathrm{kg}$, seguida de inyección de cloruro de potasio $10 \mathrm{ml}$ para inducir paro cardíaco.

En cada sitio de implantación realizamos lavado con solución salina fisiológica y empleamos $1 \mathrm{ml}$ de la solución para evaluar la fauna microbiológica mediante cultivo utilizando agar McConkey y agar sangre.

Para el análisis histológico hicimos una biopsia escisional de $2.3 \times 1.3 \times 0.5 \mathrm{~cm}$ en el sitio de implantación, seguida de fijación del material con formaldehído tamponado al $10 \%$ para laminación y análisis posterior. Las muestras se colorearon con hematoxilina/eosina y se calificaron de acuerdo con los criterios semicuantitativos y cualitativos, clasificación de la reacción inflamatoria crónica, presencia de neutrófilos, linfocitos, eosinófilos, 
fibroblastos, células gigantes, edema y neovasculogenesis 0-3 (donde 0 = ausencia de la variable evaluada, 1 = escasa presencia de la variable evaluada, 2 = presencia significativa de la variable evaluada y $3=$ alto grado de presencia de los criterios evaluados), caracterizando la presencia o no de cicatrices.

La valoración de los parámetros hematoma, hemorragia y absceso se hizo clínicamente, de forma semanal durante 2 meses, por el mismo evaluador.

\section{Resultados}

Todos los datos de este artículo fueron analizados y calculados utilizando un $95 \%$ de intervalo de con- fianza (IC). En ambos grupos, el análisis histológico pone de manifiesto una reacción inflamatoria crónica al material implantado, sin diferencia significativa entre la silicona y el PTFE (Fig. 1-3). Además, de las muchas variables analizadas en las muestras y por la evaluación clínica en el sacrificio, sólo la presencia de absceso, determinada por la presencia de fluctuación al examen clínico y salida de secreción en el momento de la retirada del implante después del sacrificio, tuvo significancia estadística ( $\mathrm{p}=\mathrm{CI} 0.01195 \%$ ), estando presente en 6 de los 10 conejos del grupo de silicona y en ninguno del grupo de PTFE. Describimos los resultados obtenidos por el análisis histológico de las muestras en la Tabla I.

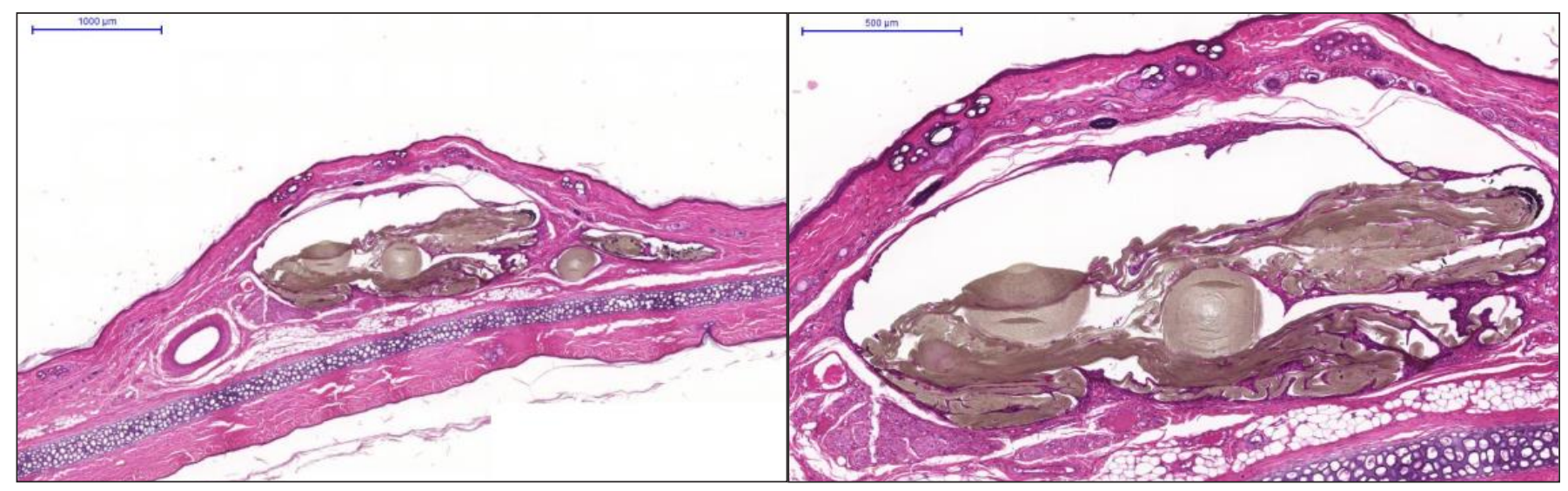

Figura 1. Fotomicrografía que muestra el implante de PTFE (H/E 5x y 20x respectivamente)

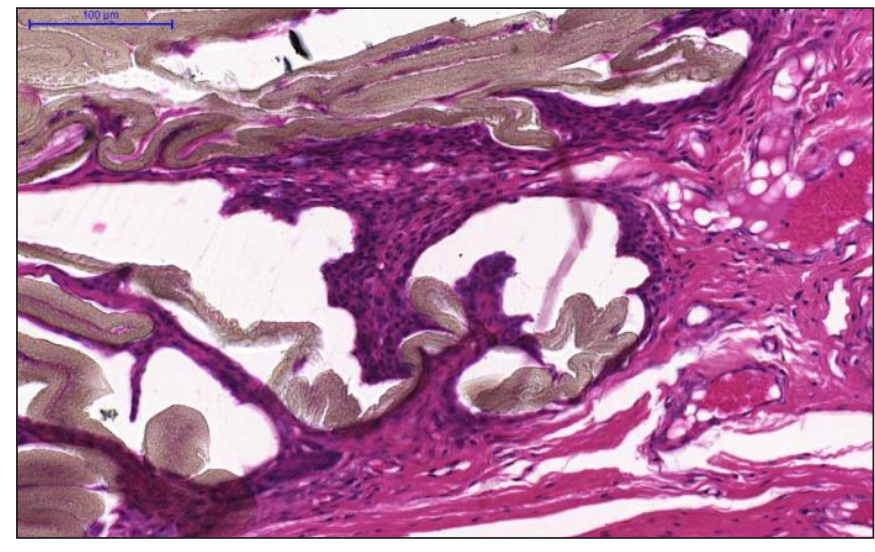

Figura 2. Fotomicrografía que muestra reacción inflamatoria crónica inducida por PTFE (H/E, 40x),
Realizamos la primera evaluación clínica de los conejos 1 semana después de los procedimientos y sus resultados están contenidos en la Tabla II. Sólo el edema $\mathrm{y}$ el hematoma demostraron diferencia significativa entre los grupos $(\mathrm{p}=0.038$, respectivamente, $95 \% \mathrm{CI}$ y $\mathrm{p}=0.001,95 \% \mathrm{CI}$ ), siendo más frecuente en el grupo de silicona. Además, la primera evaluación clínica evidenció una reacción inflamatoria aguda en el sitio de implantación (Fig. 4).
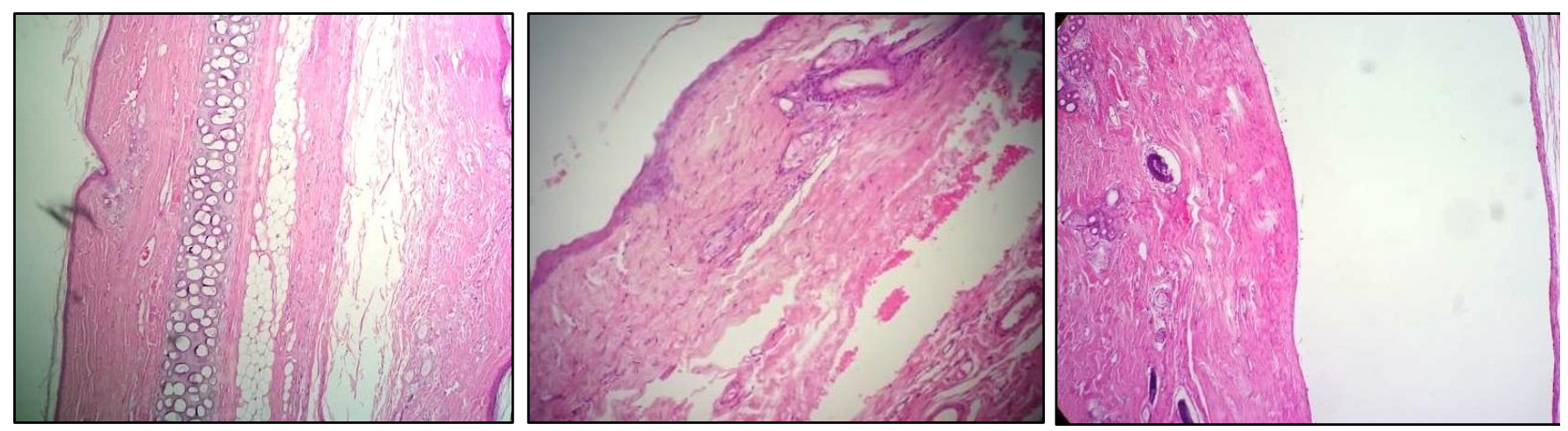

Figura 3. Fotomicrografía que muestra: A. Grupo control (H/E, 10x). B. Grupo PTFE (H/E, 16x), C. Grupo silicona (H/E, 16x). 
Tabla I. Comparación clínica entre grupos (fragmentos del silicona y PTFE) en el momento de la retirada del implante después del sacrificio.

\begin{tabular}{lccc} 
Variable & $\begin{array}{c}\text { Silicona } \\
(\mathbf{n}=\mathbf{1 0})\end{array}$ & $\begin{array}{c}\text { PTFE } \\
(\mathbf{n}=\mathbf{1 0})\end{array}$ & $\mathbf{p}^{(\mathbf{1})}$ \\
\hline Hemorragia & 2 & 1 & 1.000 \\
Cicatriz & 2 & 0 & 0.474 \\
Hematoma en el sacrificio & 2 & 0 & 0.474 \\
Absceso en el sacrificio & 6 & 0 & 0.011 \\
Cultivo positivo & 0 & 0 & 1.000 \\
Extrusión del implante & 2 & 0 & 0.474 \\
RIC* & & & \\
0 & 4 & 4 & 0.653 \\
1 & 2 & 2 & \\
2 & 1 & 3 & \\
3 & 3 & 1 &
\end{tabular}

Presencia de neutrofilos

$\begin{array}{llll}0 & 10 & 10 & 1.000\end{array}$

Presencia de linfocitos

1

2

3

Presencia de eosinófilos

0

1

Neoangiogénesis

$\begin{array}{lll}0 & 3 & 4 \\ 1 & 2 & 2 \\ 2 & 1 & 2 \\ 3 & 1 \\ 4 & 4 & 1 \\ & 0 & 1\end{array}$

Presencia de fibroblastos

$\begin{array}{lll}0 & 3 & 4 \\ 1 & 0 & 0 \\ 2 & 2 & 3 \\ 3 & 5 & 3\end{array}$

Edema

$\begin{array}{llll}0 & 9 & 8 & 1.000\end{array}$

100

2101

3 0 1

Presencia de células gigantes

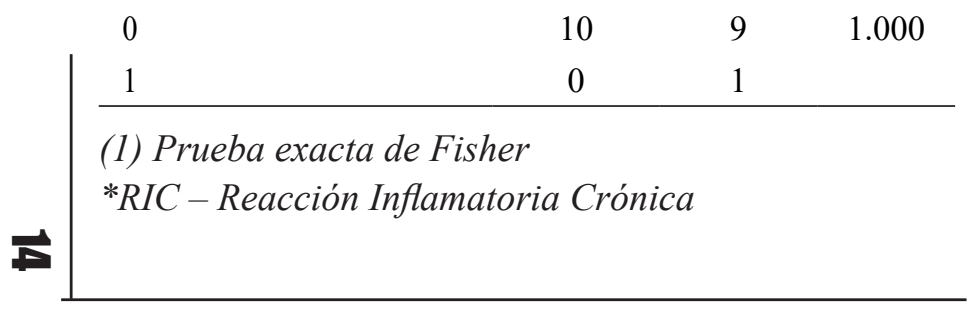

Tabla II. Comparación entre los grupos una semana después de la cirugía.

\begin{tabular}{lccc}
\hline Variable & $\begin{array}{c}\text { Silicona } \\
(\mathbf{n}=\mathbf{1 0})\end{array}$ & $\begin{array}{c}\text { PTFE } \\
(\mathbf{n}=\mathbf{1 0})\end{array}$ & $\mathbf{p}^{(\mathbf{1})}$ \\
\hline Edema & & & \\
0 & 1 & 2 & 0.038 \\
1 & 1 & 6 & \\
2 & 7 & 2 & \\
3 & 1 & 0 & \\
Hiperemia & 4 & 3 & 1.000 \\
Absceso & 0 & 0 & 1.000 \\
Adhesión & 2 & 0 & 0.474 \\
Dolor & 0 & 0 & 1.000 \\
Hematoma & & & \\
0 & 0 & 5 & 0.001 \\
1 & 1 & 4 & \\
2 & 0 & 0 & \\
3 & 4 & 0 & \\
4 & 5 & 1 & \\
\hline
\end{tabular}

(1) Prueba exacta de Fisher

Tabla III. Comparación entre los grupos 2 semanas después de la cirugía.

\begin{tabular}{|c|c|c|c|}
\hline Variable & $\begin{array}{c}\text { Silicona } \\
(n=10)\end{array}$ & $\begin{array}{l}\text { PTFE } \\
(n=10)\end{array}$ & $\mathbf{p}^{(1)}$ \\
\hline \multicolumn{4}{|l|}{ Edema } \\
\hline 0 & 6 & 9 & 0.210 \\
\hline 1 & 3 & 0 & \\
\hline 2 & 1 & 1 & \\
\hline 3 & 0 & 0 & \\
\hline Hiperemia & 7 & 6 & 1.000 \\
\hline Absceso & 2 & 3 & 1.000 \\
\hline Adhesión & 0 & 2 & 0.474 \\
\hline Dolor & 5 & 3 & 0.650 \\
\hline \multicolumn{4}{|l|}{ Hematoma } \\
\hline 0 & 3 & 9 & 0.028 \\
\hline 1 & 0 & 0 & \\
\hline 2 & 3 & 0 & \\
\hline 3 & 3 & 1 & \\
\hline 4 & 1 & 0 & \\
\hline
\end{tabular}

(1) Prueba exacta de Fisher 
Tabla IV. Comparación entre los grupos, 7 semanas después de la cirugía.

\begin{tabular}{|c|c|c|c|}
\hline Variable & $\begin{array}{c}\text { Silicona } \\
(n=10)\end{array}$ & $\begin{array}{c}\text { PTFE } \\
(n=10)\end{array}$ & $\mathbf{p}^{(1)}$ \\
\hline \multicolumn{4}{|l|}{ Edema } \\
\hline 0 & 9 & 10 & 1.000 \\
\hline 1 & 1 & 0 & \\
\hline 2 & 0 & 0 & \\
\hline 3 & 0 & 0 & \\
\hline Hiperemia & 1 & 0 & 1.000 \\
\hline Absceso & 7 & 1 & 0.020 \\
\hline Adhesión & 0 & 0 & 1.000 \\
\hline Dolor & 1 & 1 & 1.000 \\
\hline \multicolumn{4}{|l|}{ Hematoma } \\
\hline 0 & 10 & 10 & 1.000 \\
\hline 1 & 0 & 0 & \\
\hline 2 & 0 & 0 & \\
\hline 3 & 0 & 0 & \\
\hline 4 & 0 & 0 & \\
\hline
\end{tabular}

(1) Prueba exacta de Fisher

En un segundo tiempo, 2 semanas después de la cirugía, llevamos a cabo una reevaluación de los animales. En ese momento, el único parámetro que tenía diferencia significativa entre los 2 grupos fue de nuevo la prevalencia de hematoma ( $p=0.028$, CI 95\%) en el grupo de silicona. Una vez más, la evaluación clínica evidenció una reacción inflamatoria aguda en el sitio de implantación. Describimos los resultados en la Tabla III.

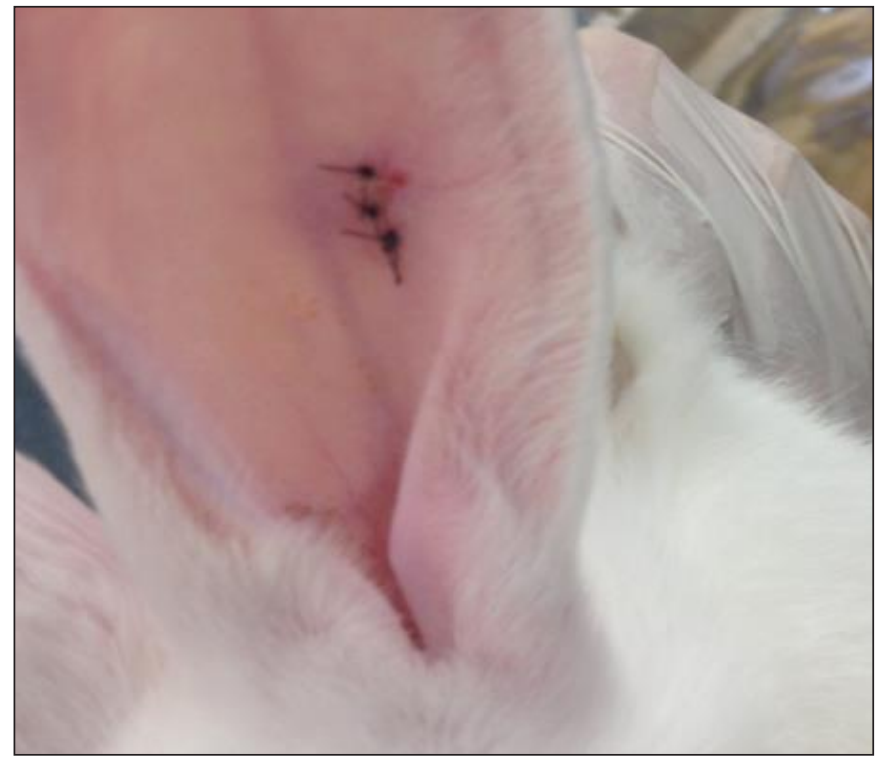

Tabla V. Comparación entre los grupos, 8 semanas después de la cirugía.

\begin{tabular}{|c|c|c|c|}
\hline Variable & $\begin{array}{c}\text { Silicona } \\
(n=10)\end{array}$ & $\begin{array}{l}\text { PTFE } \\
(n=10)\end{array}$ & $\mathbf{p}^{(1)}$ \\
\hline \multicolumn{4}{|l|}{ Edema } \\
\hline 0 & 9 & 10 & 1.000 \\
\hline 1 & 1 & 0 & \\
\hline 2 & 0 & 0 & \\
\hline 3 & 0 & 0 & \\
\hline Hiperemia & 1 & 0 & 1.000 \\
\hline Absceso & 7 & 1 & 0.020 \\
\hline Adhesión & 0 & 0 & 1.000 \\
\hline Dolor & 1 & 1 & 1.000 \\
\hline \multicolumn{4}{|l|}{ Hematoma } \\
\hline 0 & 10 & 10 & 1.000 \\
\hline 1 & 0 & 0 & \\
\hline 2 & 0 & 0 & \\
\hline 3 & 0 & 0 & \\
\hline 4 & 0 & 0 & \\
\hline
\end{tabular}

(1) Prueba exacta de Fisher

Las siguientes evaluaciones las desarrollamos a las 7 y 8 semanas de la cirugía sin encontrar discrepancia entre los grupos. Demostramos una significativa prevalencia de absceso ( $\mathrm{p}=0.02$, IC 95\%) en el grupo de silicona (7 veces mayor) y la remisión del proceso inflamatorio agudo en ambos grupos (Fig. 5). Las Tablas IV y $\mathrm{V}$ representan la tercera y cuarta evaluaciones clínicas respectivamente.

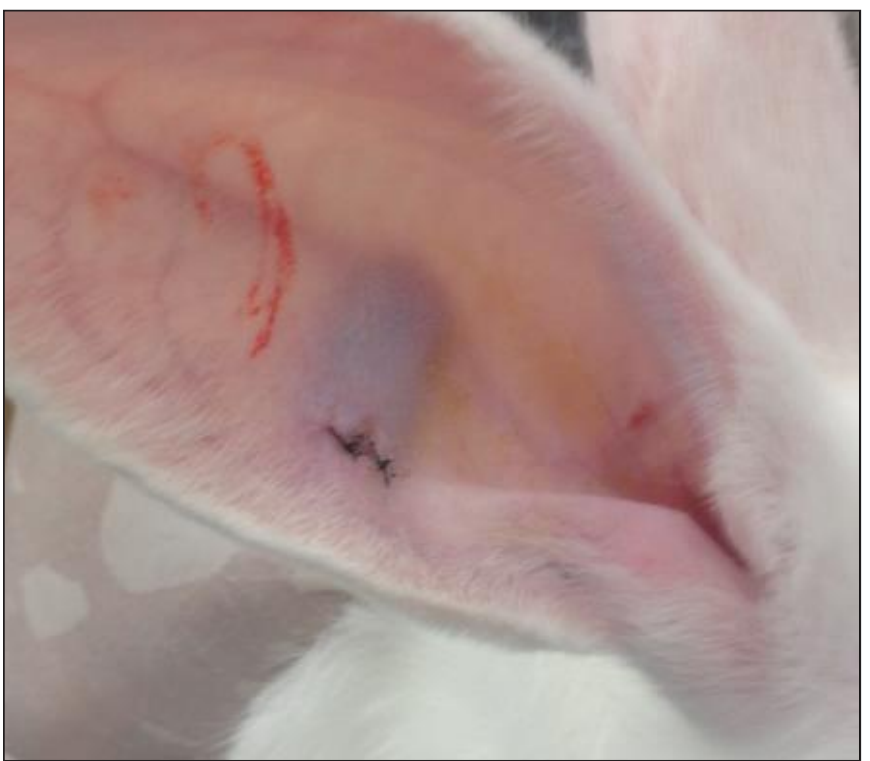

Figura 4. Sitio de implantación de silicona y PTFE una semana después del procedimiento. A. El lado de PTFE muestra señales de reacción inflamatoria aguda mínima. B. Lado de silicona que muestra señales de hematoma y reacción inflamatoria aguda. 

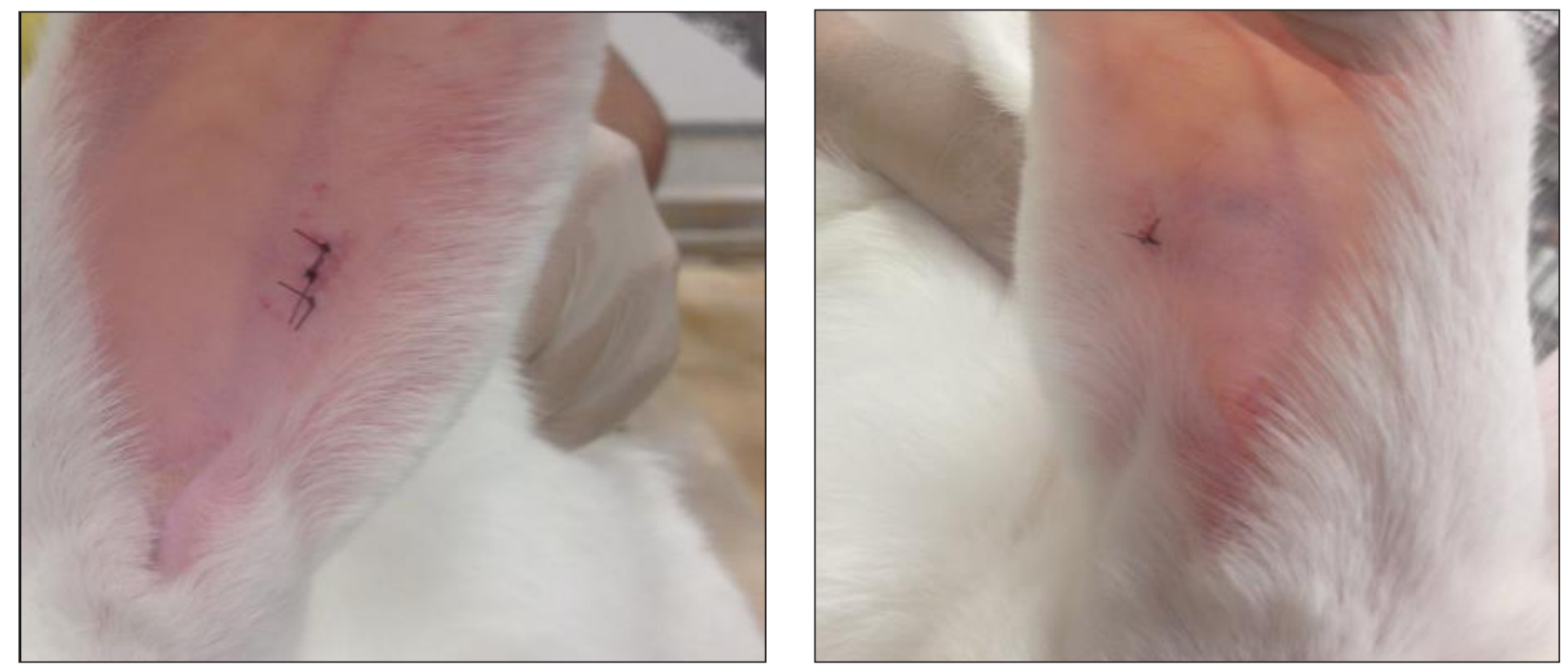

Figura 5. Sitio de implantación de silicona y PTFE al final del periodo de observación. A. El lado de PTFE no muestra señales de reacción inflamatoria aguda. B. En el lado de la silicona se ve el hematoma; no muestra señales de reacción inflamatoria aguda. Las dos fotografías son las mismas de la figura 3.

\section{Discusión}

Los implantes de silicona ofrecen un suministro casi ilimitado de material altamente biocompatible, no tóxico, fácilmente moldeable, de bajo costo y casi inerte. Por ello, desde la década de 1960 han sido uno de los materiales más utilizados en Cirugía Plástica ${ }^{(8,9)}$ Su uso tiene complicaciones, incluyendo infección, extrusión, contractura capsular, desplazamiento del implante y calcificación, todas ellas recogidas en la literatura. ${ }^{(10-12)}$ Por otra parte, la silicona no produce un buen resultado estético cuando se trata de deformidades faciales subcutáneas por ser más rígida e inflexible, dando lugar la demanda de un material diferente que no genere una gran reacción inflamatoria por el organismo y que al mismo tiempo sea más moldeable y flexible que la silicona. En el presente estudio demostramos las consecuencias inflamatorias, el edema, el hematoma y la formación de abscesos.

Con esto en mente, el PTFE, por tener una buena biocompatibilidad, moldeabilidad, ninguna propiedad alergénica, baja tasa de complicaciones, inducción de poca respuesta inflamatoria y sin formación de contractura capsular, tiene un enorme potencial para cumplir con esa demanda. ${ }^{(13-15)}$ Además, sus complicaciones son poco comunes, siendo la infección la más frecuente y rara vez se requiere la eliminación de la implante. ${ }^{(14,16,17)}$

En cuanto a las hipótesis planteadas, nuestro estudio analizó y comparó la reacción inflamatoria provocada por el PTFE y la silicona evaluando la equivalencia entre ambos cuando se trata de biocompatibilidad. El PTFE demostró reacción inflamatoria crónica con menos hematoma y absceso. Esta reacción inflamatoria crónica no tuvo diferencia estadística con la inducida por el implante de silicona ( $\mathrm{p}=0.653$, CI 95\%); sin embargo, los implantes de PTFE tuvieron una menor prevalencia de abscesos en el sacrificio ( $\mathrm{p}=\mathrm{IC} 0.011,95 \%$ ), hematoma durante las primeras 2 semanas (la primera semana $\mathrm{p}=\mathrm{IC}$ del 0.011 al $95 \%$ y la segunda semana $\mathrm{p}=\mathrm{IC}$ del 0.028 al $95 \%$ ) y edema durante la primera semana ( $\mathrm{p}=0.038$, CI 95\%).

Ambos materiales son de gran biocompatibilidad y pueden utilizarse como materia prima como implantes para la corrección de defectos faciales, teniendo el PTFE la ventaja de ser más moldeable lo que permite una mejor escultura del implante para corregir la deformidad subcutánea, siendo así un avance en la búsqueda de materiales alternativos para hacer frente a los defectos del contorno facial.

Los diferentes tamaños de los materiales de PTFE y silicona utilizados en nuestro estudio podrían ser una variable de error que condujera a menos hematoma y absceso en el grupo de PTFE, si bien estas complicaciones podrían ser también derivadas de otros factores, como la técnica quirúrgica. Como en nuestro estudio todos los procedimientos fueran realizados por el mismo cirujano y en condiciones estériles, hemos intentado minimizar así los sesgos, si bien somos conscientes de que nuestros resultados deben dar pie a futuras investigaciones.

\section{Conclusiones}

En nuestra serie experimental de estudio en conejos, el PTFE indujo reacción inflamatoria crónica al igual que la silicona pero con menos formación de absceso, hematoma y edema.

En base a este resultado creemos que el PTFE es una buena opción para uso en el tratamiento corrector de las deformidades faciales mediante implantes aloplásticos. 


\section{Agradecimientos}

AL Dr. Walter Damian Fumeketter, cirujano plástico, por su colaboración y asistencia en la traducción de este artículo.

\section{Dirección del autor}

Dr. Fausto Viterbo

UNESP Campus Botucatu

Mario Rubens Guimarães Montenegro s/n

Botucatu, São Paulo, Brasil

Correo electrónico : faustoviterbo@hotmail.com

\section{Bibliografía}

1. Herink C, Zwaka P a, Schön MP, Mempel M, Seitz CS. Serious complications following gluteal injection of silicone. Hautarzt 2013; 64: 599-602.

2. Bachynski KE. Playing hockey, riding motorcycles, and the ethics of protection. Am J Public Health 2012; 102: 2214-2220.

3. Ersek RA. Transplantation of purified autologous fat: a 3-year follow-up is disappointing. Plast Reconstr Surg. 1991; 87: 219227.

4. Panossian A GW. Polytetrafluoroethylene facial implants: 15 years later. Plast Reconstrutive Surg 2004; 113: 347-349.

5. Ugurbas SC, Kocer NE, Oto S, Kahraman B, Akova YA. Comparison of alloplast materials in experimental extraocular muscle surgery. Ophthalmic Res 2010; 44: 50-56.

6. Haddad Filho D, Zveibel DK, Alonso N GR. Comparison between textured silicone implants and those bonded with expanded polytetrafluoroethylene in rats. Acta Cir Bras 2007; 22: 187-194.
7. Serin G, Baylancicek S, Aksoy E, Polat S, Sağlican Y, Inanli S. Evaluation of tissue response to Gore-Tex (expanded polytetrafluoroethylene) implantation. J Craniofac Surg 2013; 24: $1428-1430$

8. Deva AK, Merten S, Chang L. Silicone in nasal augmentation rhinoplasty: a decade of clinical experience. Plast Reconstr Surg. 1998;102:1230-1237.

9. Zeng Y, Wu W, Yu H, Yang J, Chen G. Silicone implant in augmentation rhinoplasty. Ann Plast Surg.2002;49:495-499.

10. Graham BS, Thiringer JK, Barrett TL. Nasal tip ulceration from infection and extrusion of a nasal alloplastic implant. $J \mathrm{Am}$ Acad Dermatol. 2001;44(2 Suppl):362-364.

11. Pak MW, Chan ES, van Hasselt CA. Late complications of nasal augmentation using silicone implants. J Laryngol Otol. 1998;112:1074-1077.

12. Erlich MA, Parhiscar A. Nasal dorsal augmentation with silicone implants. Facial Plast Surg.2003;19:325-330.

13. Kim H-S, Park S-S, Kim M-H et al. Problems Associated with Alloplastic Materials in Rhinoplasty. Yonsei Medical Journal. 2014;55(6):1617-1623.

14. Godin MS, Waldman SR, Johnson CM Jr. Nasal augmentation using Gore-Tex: a 10-year experience. Arch Facial Plast Surg. 1999;1(2):118-121.

15. Jang TY, Choi JY, Jung DH, Park HJ, Lim SC. Histologic study of Gore-Tex removed after rhinoplasty. Laryngoscope. 2009;119:620-627.

16. Peled ZM, Warren AG, Johnston P, Yaremchuk MJ. The use of alloplastic materials in rhinoplasty surgery: a meta-analysis. Plast Reconstr Surg. 2008;121(3):85e-92e.

17. Conrad K, Gillman G. A 6-year experience with the use of expanded polytetrafluoroethylene in rhinoplasty. Plast Reconstr Surg. 1998;101(6):1675-1683.

18. Mendelsohn M, Dunlop G. Gore-tex augmentation grafting in rhinoplasty_ — is it safe? J Otolaryngol. 1998;27(6):337-341. 
